\title{
Effect of Pore Structure, Randomness and Size on Effective Mass Diffusivity
}

\author{
Minas M. Mezedur and Massoud Kaviany \\ Wayne Moore \\ Delphi Automotive Systems, Flint, MI 48556
}

Dept. of Mechanical Engineering, University of Michigan, Ann Arbor, MI 48109

\begin{abstract}
The effective mass diffusivity $\left\langle D_{m}\right\rangle$ of large specific surface area catalytic porous coatings is analyzed using 2-D network models of connecting arms and verified experimentally for a multiple pore length scale coating layer. The network model includes effects of variation in the lattice randomness (Voronoi tessellation in the form of Delaunay lattice triangulation), pore coordination number, pore size (Knudsen effect), and pore-size distribution on the predicted $\left\langle D_{m}\right\rangle$. The effect of pore poisoning, resulting in a pore blockage, is analyzed. Correlations for the porosity and pore-blockage dependency of $\left\langle D_{m}\right\rangle$, as well as relationships for the pore size (low-dimensionality) and multiple pore length scale effects, are also discussed. An experiment performed on a catalytic converter washcoat segment (represented by three pore length scales) placed on an otherwise impermeable wall of an electrochemical sensor shows a good agreement with the predicted $\left\langle D_{m}\right\rangle$ based on a multiple pore length scale medium with parallel diffusion paths.
\end{abstract}

\section{Introduction}

In surface mediated chemical reactions in porous media, the specific surface area $A_{s g} / V$ and the activation energy $\Delta E_{a}$ of the chemical reaction are among the parameters affecting the reaction rate. A simple, first-order relation for the volumetric consumption rate of a species $i$ is given by (Weisz, 1973)

$$
\dot{n}_{r, i}=-\frac{\left\langle u_{m}^{2}\right\rangle^{1 / 2} \rho_{i}}{4} \frac{A_{c}}{A_{s g}} \frac{A_{s g}}{V} e^{-\frac{\Delta E_{a}}{R_{g} T}}
$$

Figure 1 shows the effect of an increase in the specific surface area $A_{s g} / V$ on the activation energy $(1 \mathrm{kcal} / \mathrm{mol}=4.18$ $\times 10^{6} \mathrm{~J} / \mathrm{kmol}$ ) of a reaction that can be sustained. The results are for an average molecular weight of $M=200$ $\mathrm{kg} / \mathrm{kmol}$, characteristic of cracking of large molecular weight hydrocarbons, at $T=500^{\circ} \mathrm{C}$, and $p=1 \mathrm{~atm}$. The effect of active catalyst surface fraction $A_{c} / A_{s g}$ is also shown. The results show the need for a large specific surface area in order to achieve high-activation energy chemical reactions.

Correspondence concerning this article should be addressed to M. Kaviany.
Most porous catalysts have more than one pore length scale, resulting in a very large specific surface area. The interior surface area of the entire catalyst, however, should not be assumed to be readily accessible to the reactants. Diffusion of the reactants into these smaller pores may be the limiting factor for the overall reaction rate. Modeling of the entire catalyst treats each pore length scale separately. The effective diffusivity $\left\langle D_{m}\right\rangle$ of the reactants, starting from the largest pore length scale, can be determined for each pore length scale. Then, using the parallel diffusion model, the effect of multiple pore length scales on the effective diffusivity can be determined. The understanding of the contribution of diffusion as a limitation on the reaction rate allows such analysis to guide washcoat formulation and application and to provide more effective, economic catalysts.

Here, the fundamentals of 2-D, ordered and random lattice network modeling are used to predict the effective diffusivity of reactants and products. In the ordered lattice network models, each unit cell has a defined geometry, such as a square. In the disordered or random lattice network models, there is no set rule for the unit-cell geometry, resulting in a variable connectivity for each node. The unit-cell geometry 


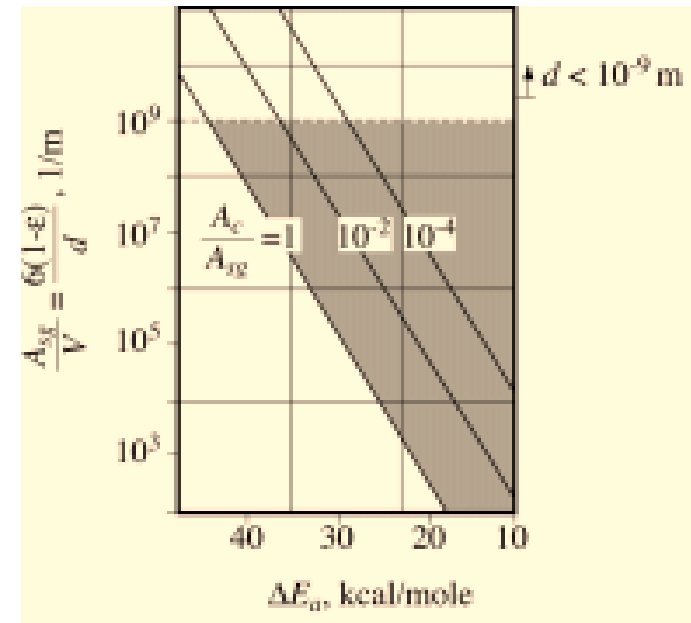

Figure 1. Effect of the specific surface area $A_{s g} / V$ on the required activation energy: (Weisz, 1973).

varies with the generation of each tessellation. Therefore, a disordered lattice network model may represent random porous media more realistically. We consider cascading catalyst pore length scales (such as in a catalytic converter washcoat), each having different geometric properties such as porosity, pore size, pore-size distribution, and pore connectivity. The effect of pore-size distribution is represented by the arm thickness $d$ having a probability distribution function $P(d)$. The influence of such geometric properties on the effective diffusivity is determined assuming no reaction occurs on the pore surface. The predictions are compared to an analytical result obtained for an ordered lattice network model. Through correlations and relationships, the dependency of $\left\langle D_{m}\right\rangle$ on the porosity, pore size (low dimensionality), and presence of multiple pore length scales, is predicted. Moreover, the effect of catalyst poisoning, that is, pore blockage, on the effective diffusivity is analyzed.

A limiting-current zirconia oxygen sensor with a gas diffusion barrier is designed to measure the oxygen effective diffusivity in a $\mathrm{O}_{2}-\mathrm{N}_{2}$ gas mixture. The results are compared with the predictions.

\section{Ordered and Random Network Models}

Two-dimensional (2-D) pore network models have been used to predict the effective mass diffusivity $\left\langle D_{m}\right\rangle$ in porous media. This is done by treating the pores as a network of regular, rectangular arms (Mann et al., 1986). Each such arm connects to the adjacent nodes, and, by changing the node coordination number, tetragonal and hexagonal networks are generated. Each node has different geometric characteristics, while the connectivity remains uniform for the network. Figures $2 \mathrm{a}$ and $2 \mathrm{~b}$ show the location of the nodes for an ordered and a random tetragonal network (generated by allowing each node to be placed at a prescribed periodic or random location within the square, ordered lattice). Although both network models are generated on an ordered lattice, the ordered tetragonal network model creates the least tortuous diffusion path, that is, the shortest distance between any two

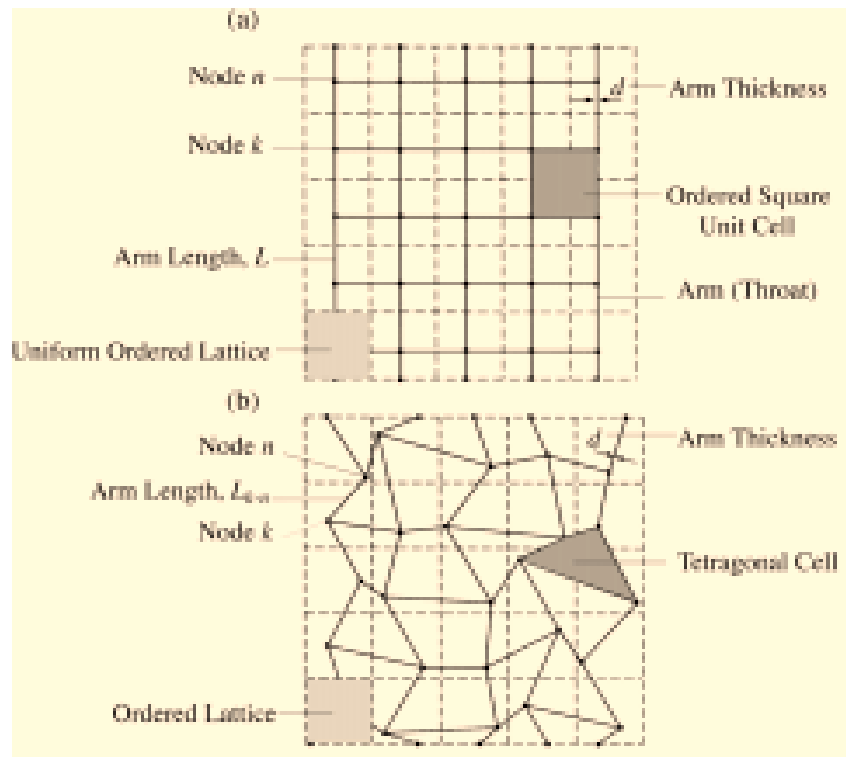

Figure 2. Location of nodes for (a) a square and (b) random tetragonal network.

nodes. To represent the randomness of real porous media, we introduce arm length and lattice randomness. This is done by using a random tetragonal network (ordered lattice, random arm length). The disordered lattice is discussed later in this section. Here, by connecting each node to both horizontal and vertical adjacent nodes, the arm length is determined and the arm thickness $d$ is assigned using the pore-size distribution $P(d)$ and the average porosity $\epsilon$.

The mass-transfer rate of species $i$ from node $k$ to $n$ (shown in Figure 2) is determined using the Fick Law for 1-D steady-state diffusion, that is

$$
\dot{M}_{i, k-n}=-A D_{m, i} \frac{\left(\rho_{i, k}-\rho_{i, n}\right)}{L} .
$$

Then, for each node $k$, the species mass balance is applied. To determine the species density at each node $\rho_{i, k}$ and the species mass-flow rate for each arm $\dot{M}_{i, k-n}$, a set of algebraic equations is solved. The mass-flow rates are integrated over the lower (and upper) boundary to determine the total massflow rate. Then, the effective mass diffusivity is determined from the local volume averaging theory as (Kaviany, 1995)

$$
\left\langle\dot{\boldsymbol{m}}_{i}\right\rangle=-\left\langle D_{m, i}\right\rangle \nabla\left\langle\rho_{i}\right\rangle
$$

where an isotropic effective mass diffusivity $\left\langle D_{m, i}\right\rangle$ is assumed. This effective diffusivity is normalized using the bulk diffusivity $D_{m, i}$ and its variation with respect to the porosity, pore structure, and so on, is determined.

The binary diffusion coefficient is determined from the kinetic theory (for low pressures) as (Bird et al., 1960)

$\frac{p D_{m, i-j}}{\left(p_{c, i} p_{e, j}\right)^{1 / 3}\left(T_{c, i} T_{c, j}\right)^{5 / 12}\left(1 / M_{i}+1 / M_{j}\right)^{1 / 2}}=a\left[\frac{T}{\left(T_{c, i} T_{c, j}\right)^{1 / 2}}\right]^{b}$, 


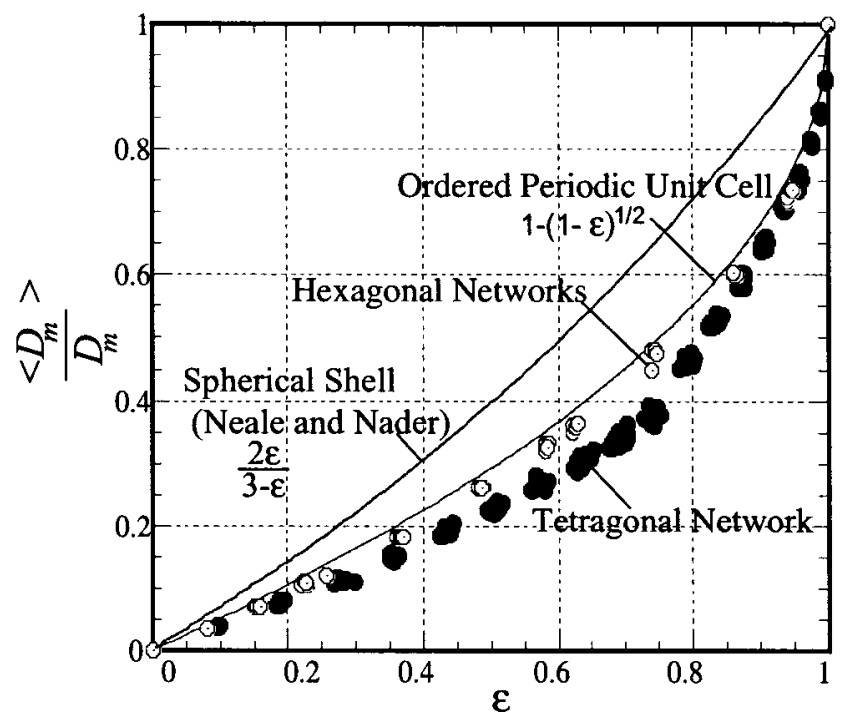

Figure 3. Variation of the effective diffusivity with respect to porosity for the tetragonal and hexagonal network models.

and the bulk diffusivity of a species $i$ into a stagnant mixture is determined from

$$
D_{m, i}=\frac{1}{1-\left(\rho_{i} / \rho\right)} \sum_{j=1}^{n} D_{m, i-j}\left(\rho_{j} / \rho\right) .
$$

In Eq. $4, a$ and $b$ are constants depending on the specific, nonpolar gas pairs.

\section{Coordination number}

Using the network model for a 2-D, isotropic periodic unit-cell geometry, that is, an ordered tetragonal network model, the effective diffusivity is related to the bulk diffusivity and porosity through (the heat-transfer analog is given in Kaviany (2001))

$$
\frac{\left\langle D_{m, i}\right\rangle}{D_{m, i}}=\frac{\left\langle D_{m}\right\rangle}{D_{m}}=1-(1-\epsilon)^{1 / 2} .
$$

Figure 3 shows the variations in the effective mass diffusivity with respect to the porosity for the tetragonal and hexagonal network models. The above result for the isotropic, periodic unit-cell geometry and the result of Neale and Nader (1973) are also shown for comparison. The random network model predicts a lower effective mass diffusivity for a given porosity, indicating that the randomness results in a larger resistance, that is, more tortuous mass transport paths. This difference can also be noted by comparing the ordered square unit cell (Figure 2a) and the tetragonal network models (Figure $2 b$ ), in which the total arm length between vertical boundaries is much larger than that of the square unit cell. Note that since a 2-D geometry is used, $d$ stands for the arm width or thickness and $L$ for the arm length. Moreover, the hexagonal network model predicts a higher effective diffusiv- (a)

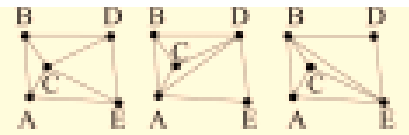

(b)

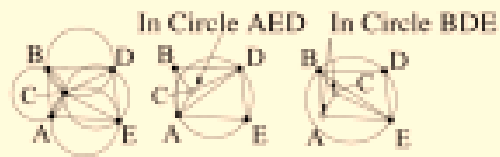

Ordered Lattice

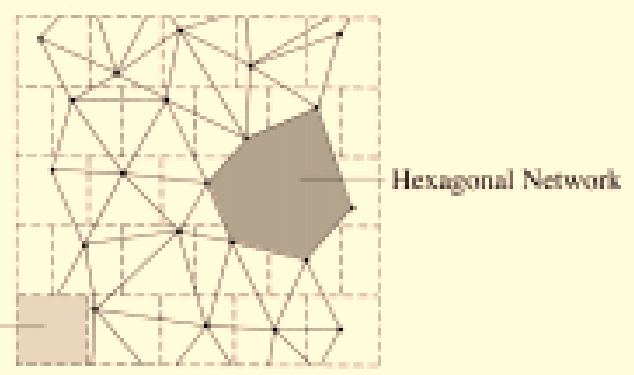

(d)

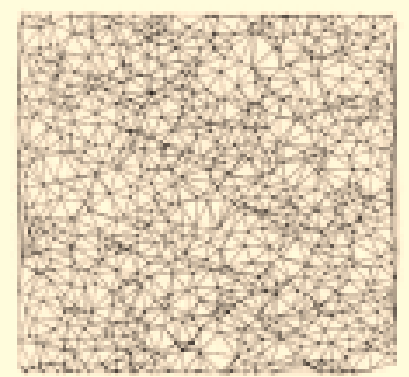

Figure 4. (a) Three possible triangulations for five set of points; (b) application of the Delaunay triangulation rule to each set of five points, and location of nodes; for (c) random tetragonal network; and (d) Delaunay triangulation.

ity. This is expected, since diffusion is less resistive through a medium having a larger pore connectivity.

\section{Lattice randomness}

The tetragonal and hexagonal network models have some geometric limitations such as having defined ordered lattices (each node has its unit cell), and this results in limiting the tortuosity of the model. This limitation can be overcome to some extent by using a network model generated by the Delaunay triangulation, a subset of Voronoi polygons and Dirichlet tesselation (Ohya et al., 1984). In this model, the locations of the nodes are determined randomly. Among lines connecting adjacent nodes, $\mathrm{AB}$ becomes an arm of the Delaunay triangulation if there is a circle passing through nodes $\mathrm{A}$ and $\mathrm{B}$ such that all points other than $\mathrm{A}$ or $\mathrm{B}$ lie outside the circle. Figure $4 \mathrm{a}$ shows three possible triangulations for a five-point set. Only the first configuration obeys the Delaunay triangulation rule, since the circles drawn for AED and BDE will not be empty, as shown in Figure 4b. These properties of the Delaunay triangulation, that is, random node locations and a more sophisticated rule for selecting the neigh- 
boring nodes, increase the randomness of the network. An intrinsic Matlab function is used to generate the Delaunay triangulation. Since the nodes are generated randomly, the triangulation is spatially uniform. Arm lengths (each side of the triangle) are the result of the tessellation and cannot be changed. However, each arm thickness can be assigned using a size distribution function $P(d)$. The mass-transfer rate of species $i$ from node $k$ to $n$ is determined using the Fick Law and then the effective diffusivity is determined using Eq. 3. Although the coordination number is a variable for each node, it follows from the Euler theorem that the average number of connections for each node is equal to six (Ohya et al., 1984). This nature of the Delaunay triangulation enables us to compare the predictions with those from the hexagonal network model shown in Figure 4c. The network node-number sensitivity is determined by changing the node numbers from $2 \times 2$ to $45 \times 45$. The results show that $33 \times 33$ node network is large enough to eliminate the node-number dependence. Figure $4 d$ shows a Delaunay triangulation with a $33 \times 33$ node network in which the random lattice structure is evident.

Computations for both the hexagonal network model and the Delaunay triangulation network are done on network models of $33 \times 33$ nodes. Figure 5 shows the variation in the effective mass diffusivity with respect to porosity for the hexagonal network model and for the Delaunay triangulation network model. The lower effective diffusivity predicted by the Delaunay triangulation indicates that more resistive paths are generated by this method. However, if this is compared with the tetragonal network predictions, the Delaunay triangulation predicts a higher effective diffusivity (as a result of higher connectivity). When compared to the hexagonal network model, the Delaunay triangulation gives a slightly lower effective diffusivity because it results in an average arm length that is larger than that in the hexagonal network model.

\section{Pore-size randomness}

Most porous media have a nonuniform pore-size (or arm thickness) distribution. Thus, the inclusion of the pore-size distribution into the network model results in a more realistic prediction of the effective diffusivity for the porous medium.

In the network model, the arm thickness is assigned to each pore randomly using a probability density function $P(d)$. To show the effect of the arm thickness distribution on the effective diffusivity, computations for uniform and normal probability density functions are made. No correlation has been assumed between the different arm thicknesses, that is, there is no preference (or limitation) in assigning an arm thickness based on the thickness assigned to the neighboring arms. A uniform probability density function creates arm thicknesses having the same thickness probability for each arm, while, for the normal probability density function, the arm thickness probability differs for each arm. Figure 6 shows the effective mass diffusivity predictions for the uniform and normal probability density functions. It can be seen that the uniform probability density function predicts a lower effective mass diffusivity value, setting the lower limit for predictions. This is because the probability of a thinner arm to be connected to a thicker arm is high, resulting in a higher mass-transfer resistance.

\section{Pore-Size (Knudsen Number) Effect}

When the arm thickness is small compared to the mean-free path of the molecules, molecules collide with the arm surface with increasingly higher probability (compared to intermolecular collisions). This is is demonstrated by the magnitude of the Knudsen number, defined as (Kaviany, 1995)

$$
\begin{gathered}
\mathrm{Kn}_{d}=\frac{\lambda_{m}}{d} \\
\lambda_{m}=\frac{1}{2^{1 / 2} \pi} \frac{k_{B} T}{d_{m}^{2} p} \\
p=\frac{R_{g}}{M} \rho T,
\end{gathered}
$$

where $M$ is the mixture molecular weight.

For $\mathrm{Kn}_{d}>0$, the Knudsen diffusion $D_{\mathrm{K}}$ becomes significant, while for $\mathrm{Kn}_{d}<0.1$, the bulk diffusion dominates. For oxygen, using $d_{m}=3.61 \times 10^{-10} \mathrm{~m}, p=1.013 \times 10^{5} \mathrm{~Pa}$, and $T=600 \mathrm{~K}$ results in $\lambda_{m}=143 \mathrm{~nm}$. The expression for the Knudsen diffusivity $D_{\mathrm{K}}$, as a function of the arm thickness, is obtained by starting from the conservation of momentum in a capillary arm, and is estimated as (Cunningham and Williams, 1980; Kast and Hohenthanner, 2000)

$$
D_{\mathrm{K}}=\frac{4}{3}\left(\frac{R_{g} T}{2 \pi M}\right)^{1 / 2} d
$$

Here, we apply this relation to the 2-D transport, that is, no curvature in the arm geometry. The mass diffusivity $D_{m}^{\prime}$ is defined in terms of the bulk $D_{m}$ and the Knudsen $D_{k}$ diffusions as

$$
\frac{1}{D_{m}^{\prime}} \equiv \frac{1}{D_{m}}+\frac{1}{D_{\mathrm{K}}} .
$$

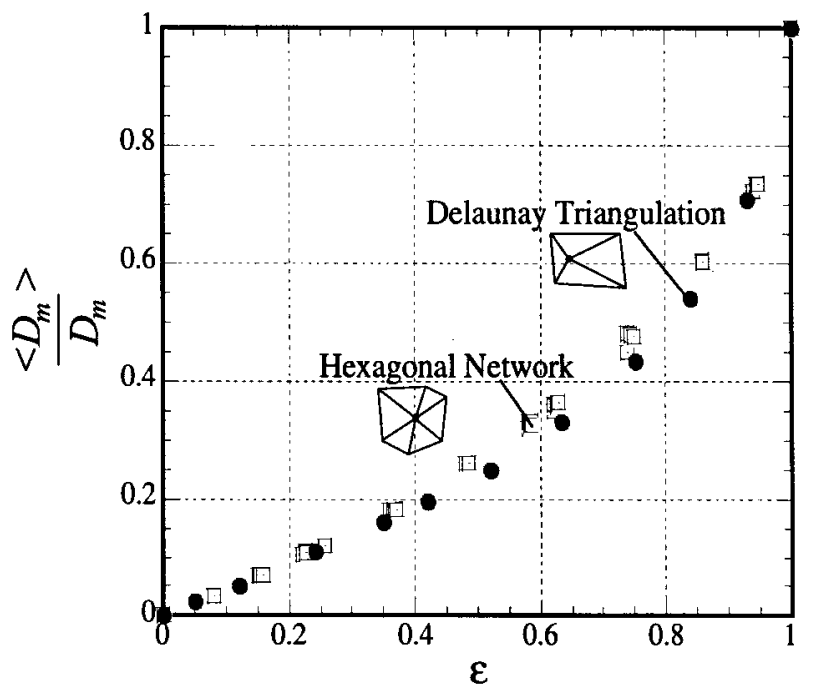

Figure 5. Variation in the effective diffusivity with respect to porosity for the hexagonal network model and the Delaunay triangulation. 


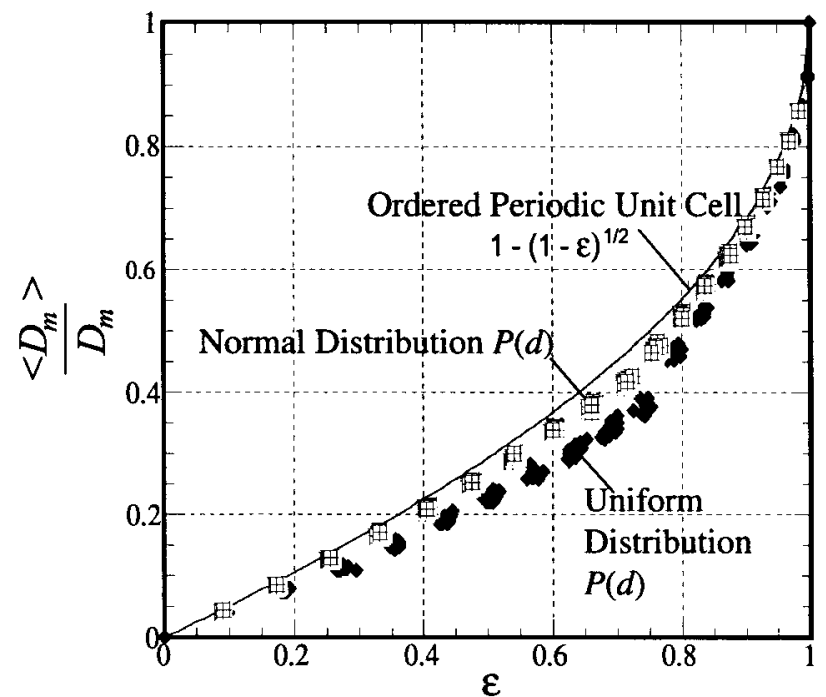

Figure 6. Variation of predicted effective diffusivity with respect to porosity for uniform and normal pore-width density functions $P(d)$.

By using Eqs. 2, 10, and 11, the variation of the oxygen mass diffusivity with respect to the arm thickness $d$ (for a given temperature, pressure, and a binary $\mathrm{O}_{2}-\mathrm{N}_{2}$ gas mixture) is shown in Figure 7. Figure 7 demonstrates the significance of the Knudsen number, representing the various length scales in a multilength scale porous medium. Three different diffusion regimes are identified, depending on the pore size. When the average pore size is of the order of the molecular diameter, the diffusivity should be determined from a molecular configurational model (Weisz, 1973), in which the pore crys-

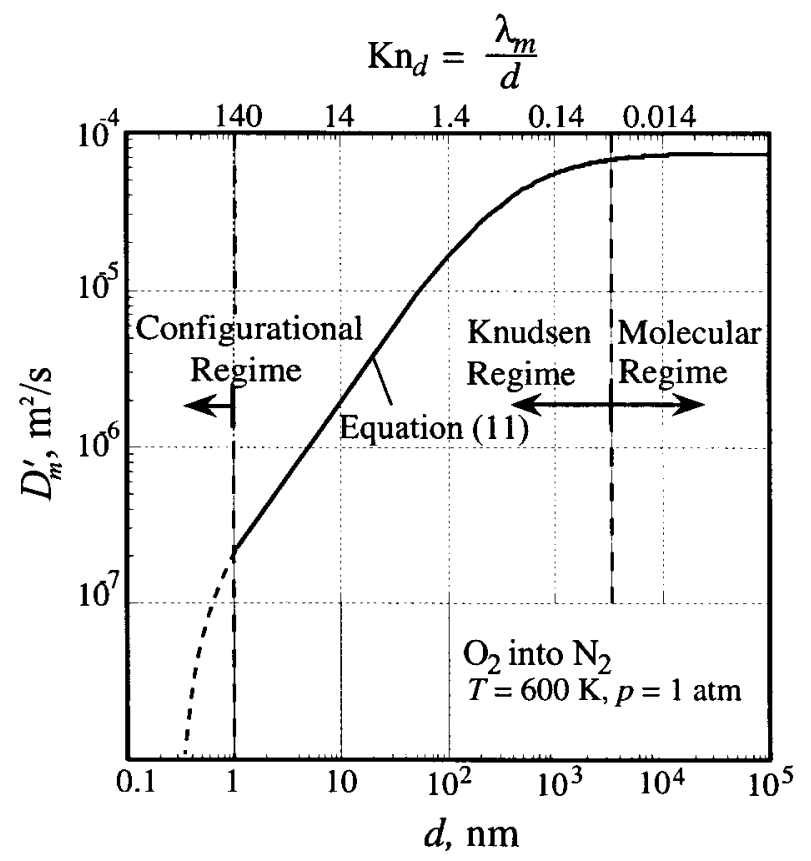

Figure 7. Variation in the mass diffusivity with respect to pore size (or Knudsen number). talline structure and geometry, the molecular dimension of diffusing species, and the pore-size distribution all become important.

\section{Pore Blockage Effect}

Deposition of lead, phosphorus, silicone, sulfur and their compounds on the pore surface poisons the catalytic converter by blocking the pore entrance. This effect is included in the network model to obtain a realistic prediction for the effective diffusivity. In the ordered lattice, random tetragonal model, randomly generated poisonous contaminants having circular geometry are assigned to each unit cell of the network model. Then, the distances from each blockage area to adjacent arms are determined, and if these intersect with an arm, the arm is assumed to be partly or totally blocked. From the dimensions of the blockage area and the arm, the part of the arm occupied by the contaminant is determined and a new arm thickness is assigned. The dimensions of the arms having no contaminant remain the same. Figures $8 \mathrm{a}$ and $8 \mathrm{~b}$ show the presence of the contaminants and their effect on the effective diffusivity in a random tetragonal network model. Since the blockage areas are generated randomly, they may not block any arms, that is, they may be present only in the solid phase. The ratio of the contaminant volume to the arm volume is designated as $V_{b} / V_{p}$. Figure $8 \mathrm{~b}$ shows that as $V_{b} / V_{p}$ increases, the effective diffusivity decreases.

\section{Example of Catalytic Coating}

The coated porous layer in a catalytic converter, where the surface-mediated chemical reactions occur, is referred to as the washcoat. This layer is a mixture of precious metals, alumina, binders, and stabilizers. It provides a very large specific surface area $A_{s g} / V$ for these chemical reactions. The washcoat is a highly porous layer having different pore-size distributions at different pore length scales. Figures 9a, 9b, and 9c show SEM images of three different washcoat length scales. As shown, each of the length scales has a different pore-size distribution and porosity.

The pore-size distribution for each length scale is obtained using the image processing application software, NIH-Image 1.61 (available at http://rsb.info.nih.gov/nih-image/). The program is capable of determining the geometric characteristics of objects (in this case, pores) within a desired region. It can generate a probability density distribution $P(d)$ for the object in this region and this is then used here to generate arm thicknesses for the 2-D network models. From the geometric characteristics, the total pore area is computed for each length scale. Dividing the total pore area by the total area, a porosity $\epsilon_{i}$ associated with each length scale is determined. Figures $10 \mathrm{a}, 10 \mathrm{~b}$, and $10 \mathrm{c}$ show the pore-size distributions obtained from the SEM images shown in Figures 9a, 9b, and $9 \mathrm{c}$. The average pore size $\left\langle d_{i}\right\rangle$ and porosity $\epsilon_{i}$ for the three length scales shown in these SEM images are: $\left\langle d_{1}\right\rangle=13$, $\left\langle d_{2}\right\rangle=0.82$, and $\left\langle d_{3}\right\rangle=0.10 \mu \mathrm{m}$, and $\epsilon_{1}=0.21, \epsilon_{2}=0.34$, and $\epsilon_{3}=0.28$, respectively.

Using the results for these length scales, the 2-D random tetragonal network model is used to predict the effect of the arm thickness (here, the pore size is used for arm thickness) for these length scales. The results are shown in Figure 11. 

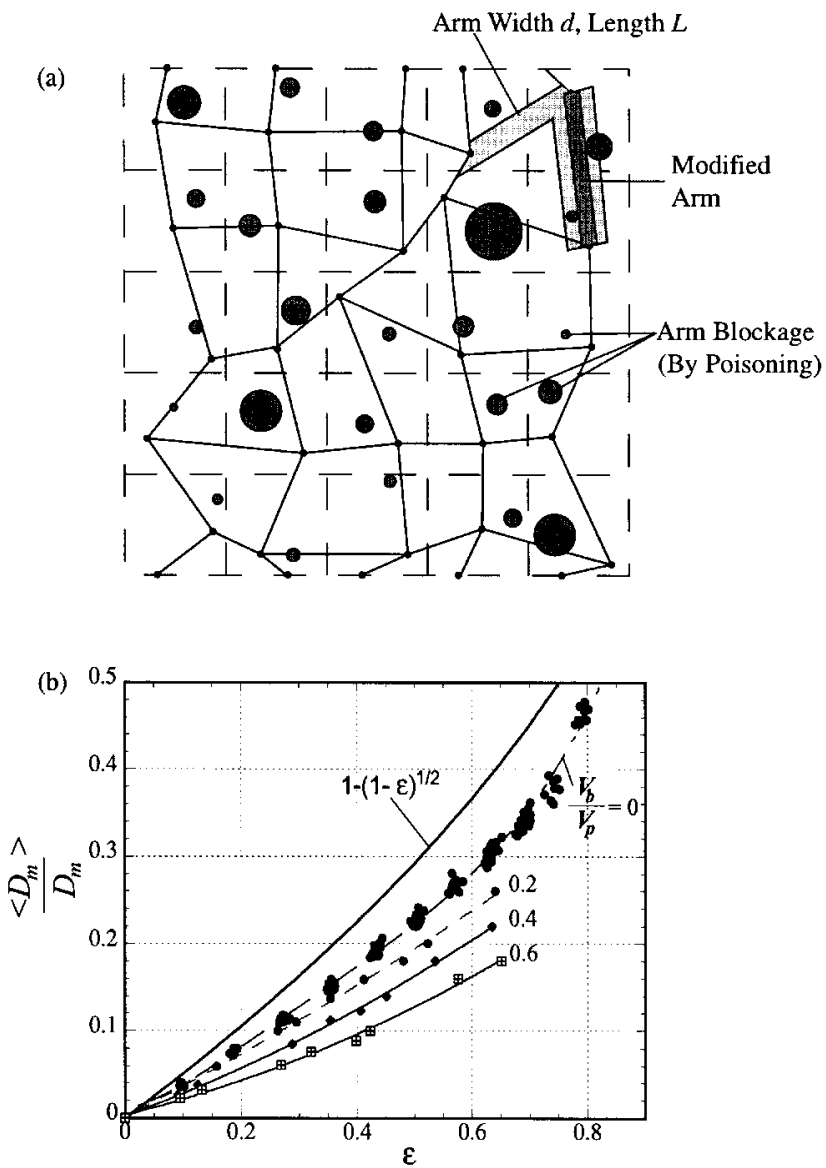

Figure 8. (a) Rendering of the presence of a blocking contaminant in pore volumes; (b) predicted effect of the poisonous pore-blockage contaminant on effective diffusivity.

The Knudsen effect becomes more significant for the smallest length scale $\left\langle d_{3}\right\rangle$, as shown in Figure 11.

\section{Measurement of Effective Mass Diffusivity}

Effective mass diffusivity can be measured using a zirconia oxygen sensor. Since yttria stabilized zirconia (YSZ) is a good oxygen ion conductor, it can be used as a solid electrolyte. Figure 12a shows the physical principle of a conventional oxygen sensor. In the conventional oxygen sensor, the oxygen concentration is determined using zirconia as a solid electrolyte, that is, ion conductor. This is done by exposing one side of the sensor to a gas stream with the oxygen concentration to be determined, and by using air as a reference gas with a known oxygen concentration $\rho_{\mathrm{O}_{2}}$. Due to the difference in the oxygen partial pressure on the two sides of the sensor, oxygen ions migrate from the oxygen rich side, that is, air, to the oxygen lean side. This ion current results in an electric potential difference $\Delta \varphi$ across the electrodes. This potential difference is the output of a conventional oxygen sensor and is given by (Kim et al., 1996)

$$
\Delta \varphi=\frac{R_{g} T}{4 F} \ln \left(\frac{p_{\mathrm{O}_{2,1}}}{p_{\mathrm{O}_{2, \infty}}}\right) .
$$

(a) Largest Pore Length Scale, $\left\langle d_{1}\right\rangle$

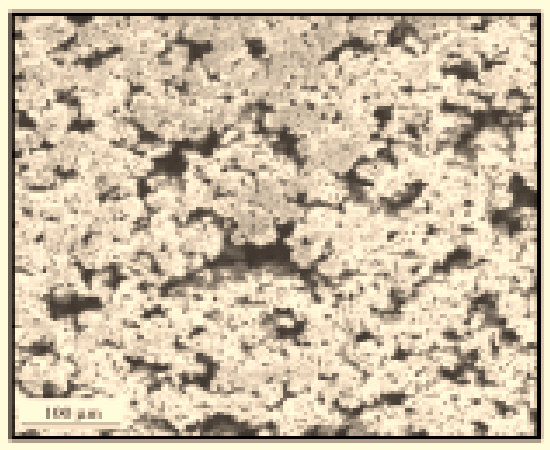

(b) Intermediate Pore Length Scale, $\left\langle d_{2}\right\rangle$

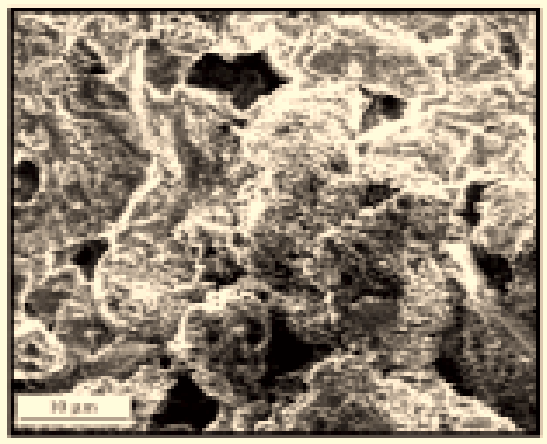

(c) Smallest Pore Length Scale, $\left\langle d_{3}\right\rangle$

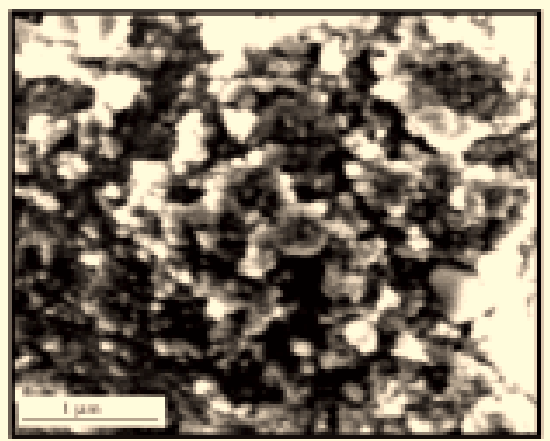

Figure 9. Automobile catalytic converter washcoat at different magnifications, showing different pore length scales and porosities.

The limiting-current oxygen sensor also uses zirconia as the solid electrolyte, but uses an opposite direction for the ion flow (Lee et al., 1996). Figure 12b shows a limiting-current oxygen sensor. Here, in contrast to the conventional oxygen sensor, an electric potential difference is applied to the electrodes, and, as a result, oxygen ions migrate to the air side. This is referred to as oxygen pumping and its magnitude is detected as the electric current $J_{e}$. Without a diffusion barrier (the barrier is shown in Figure 12b), the pumping current increases linearly with the amount of voltage applied. The current is saturated when using a diffusion barrier placed on the gas-side electrode to control the oxygen diffusion rate. 
(a) Largest Pore Length Scale

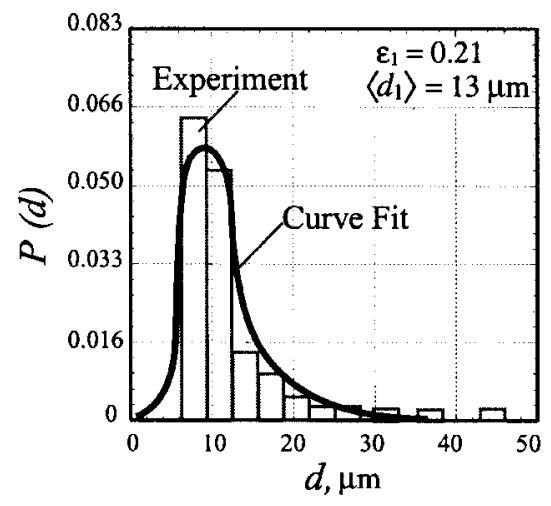

(b) Intermediate Pore Length Scale

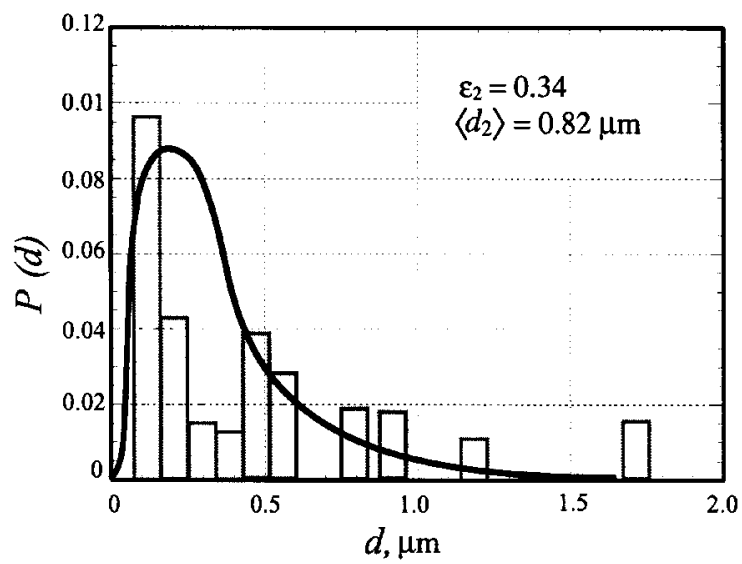

(c) Smallest Pore Length Scale

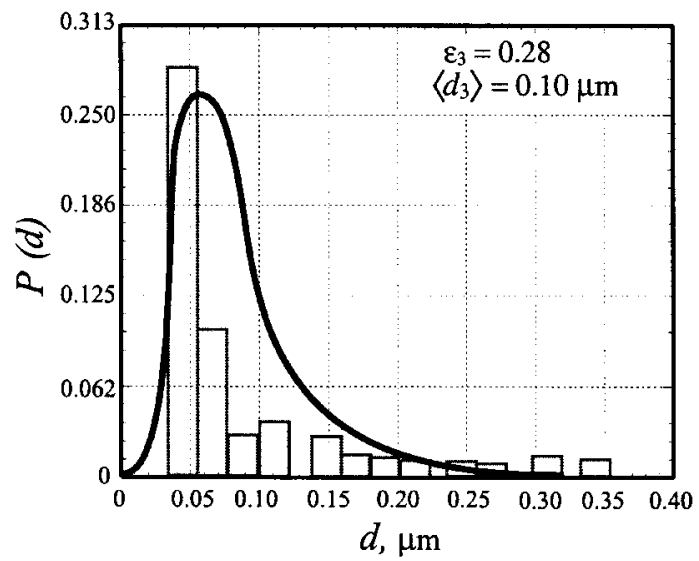

Figure 10. Pore-size distributions associated with different pore length scales in the washcoat.

The anticipated current-voltage output for the limiting-current oxygen sensor is shown in Figure 12c, where the saturation in the current, due to the diffusion barrier, is shown. This saturation current is related to the gas-side oxygen partial pressure. The slope of the line depends on the pumping capacity of the electrolyte, which results from the electrical resistance of the zirconia. The saturation current depends on the oxygen diffusion rate across the diffusion barrier, and is

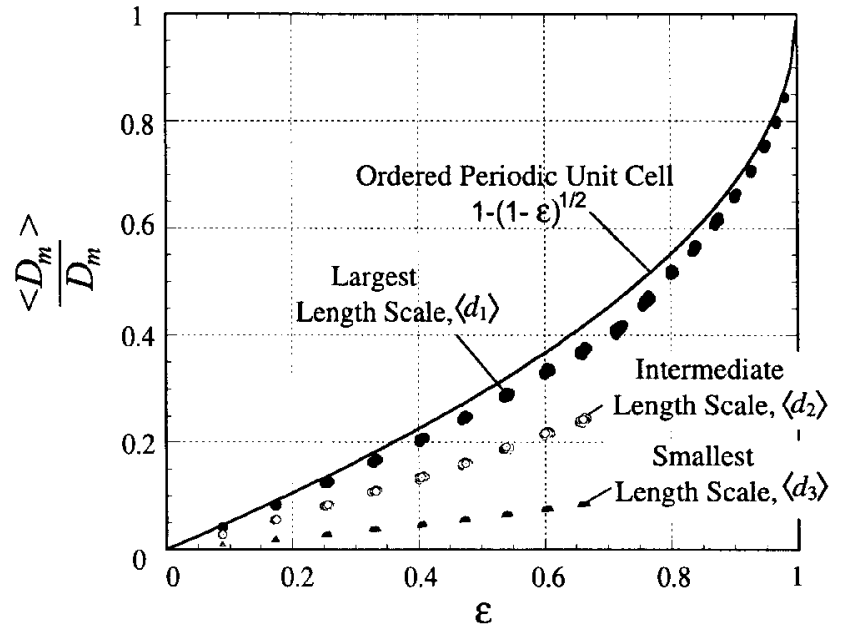

Figure 11. Predicted effect of the pore length scale on the variation of the effective mass diffusivity with respect to porosity.

determined from

$$
J_{e}=4 F \frac{\dot{M}_{\mathrm{O}_{2}}}{M_{\mathrm{O}_{2}}}
$$

Using the Fick Law for a 1-D, steady-state diffusion, we have

$$
\dot{M}_{\mathrm{O}_{2}}=\frac{\left\langle D_{m}\right\rangle A}{L}\left(\rho_{\mathrm{O}_{2, \infty}}-p_{\mathrm{O}_{2, s}}\right) \text {. }
$$

Using the measured current and assuming that the oxygen partial pressure at electrode 1 is zero (due to the diffusioncontrolled reaction rate at the catalytic surface), the effective diffusivity of oxygen for the porous medium is determined from Eqs. 13 and 14 as

$$
J_{e}=\frac{4,000 F\left\langle D_{m}\right\rangle A}{M_{\mathrm{O}_{2}} L} \rho_{\mathrm{O}_{2, \infty}} .
$$

The measurement device is shown in Figure 13. A zirconia solid electrolyte element is used as the oxygen ion pump. The platinum electrodes on the electrolyte are plasma coated. The element is placed in a stainless-steel housing. Sealing is achieved using the talc powder. A pre-formed talc powder tablet is compressed by an alumina ring using a high pressing force. Under this applied force, the talc powder tablet flows, filling the possible gap spaces and preventing any air leakages. A leakage test was conducted with no opening (hole) on the housing, that is, having no connectivity between the electrode and the gas-side oxygen. An insignificant signal is detected indicating a good seal around the reference air side. The calibration of the sensor was then performed using a well-defined opening $(\epsilon=1)$ as the diffusion barrier. The oxygen molecules on the gas side diffuse through this opening when a voltage is applied. Since the opening is small, the diffusion through it limits the sensing current. Using Eq. 15, 
(a) Conventional Oxygen Sensor

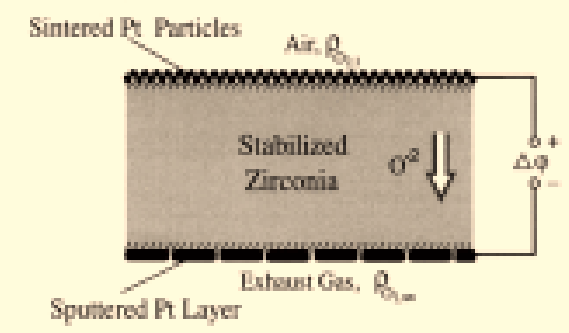

(b) Limiting-Current Oxygen Sensor

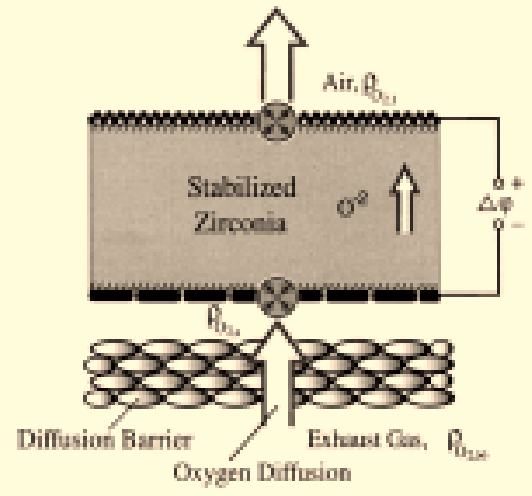

(c) Saturation Current for Different Oxygen Mass Fractions

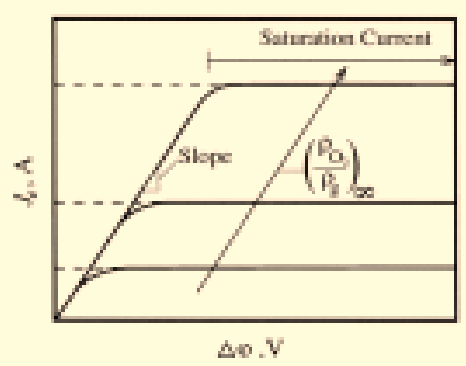

Figure 12. (a) Physical principle of a conventional oxygen sensor; (b) limiting-current oxygen sensor; (c) anticipated voltage-current output of the limiting-current oxygen sensor.

the molecular (or bulk, $\epsilon=1$ ) diffusivity is determined. Finally, the opening was filled with a washcoat slurry and the effective diffusivity was measured for $\epsilon<1$. This was done by dipping the sensor housing into a prepared washcoat slurry.

\section{Results and Discussion}

The predictions from the 2-D network models indicate that the washcoat geometric properties, that is, porosity, pore size, pore-size distribution, pore connectivity, and pore blockage, all affect the effective diffusivity $\left\langle D_{m}\right\rangle$. The effect of the pore size, that is, low dimensionality, is included in $D_{m}^{\prime}$ through Eq. 11. The results show that the ordered lattice, random tetragonal network with a uniform arm thickness distribution results in the lowest effective diffusivity (however, this value is within $20 \%$ of that of other distributions and network models). Since the influences of the coordination number, lattice regularity, and randomness are not dominant, these effects can be summarized by using a representative limit: the tetragonal network model with a normal arm thickness distri- bution. These representative results are curve fitted, and the effects of porosity $\epsilon$ and pore blockage $V_{b} / V_{p}$, for $0 \leq \epsilon \leq 0.65$ and $0 \leq V_{b} / V_{p} \leq 0.6$ are correlated as

$$
\frac{\left\langle D_{m}\right\rangle}{D_{m}^{\prime}}=\left[1-(1-\epsilon)^{0.46}\right]\left[\left(1-\frac{V_{b}}{V_{p}}\right)^{0.71}\right] \text {. }
$$

In the washcoat used in the experiment (example of catalytic coating), there are three distinct length scales, each having porosity $\epsilon_{i}$. In the experiment, the saturation currents for an open hole $\left(\epsilon_{1}=1\right)$ and for a washcoat layer placed in the hole $\left(\epsilon_{1}<1\right)$, are measured. Table 1 lists the magnitude of the parameters and the measured quantities. The measured saturation current is plotted with respect to far-field oxygen mass fraction $\left(\rho_{\mathrm{O}_{2}} / \rho_{g}\right)_{\infty}$ in Figure 14 .

Since the washcoat has three pore length scales, using a parallel resistance arrangement, we write the effective diffusivity $\left\langle D_{m}\right\rangle$ as

$$
\begin{aligned}
& \left\langle D_{m}\right\rangle=D_{m, 1}^{\prime}\left[1-\left(1-\epsilon_{1}\right)^{0.46}\right] \\
& +D_{m, 2}^{\prime}\left[1-\left(1-\epsilon_{2}\right)^{0.46}\right]\left(1-\epsilon_{1}\right) \\
& +D_{m, 3}^{\prime}\left[1-\left(1-\epsilon_{3}\right)^{0.46}\right]\left(1-\epsilon_{1}\right)\left(1-\epsilon_{2}\right)+\ldots
\end{aligned}
$$

where $D_{\mathrm{m}, 1}^{\prime}, D_{m, 2}^{\prime}$, and $D_{m, 3}^{\prime}$ are determined using Eq. 11 using the arm-thickness effect for each pore length scale, and the porosities $\epsilon_{1}, \epsilon_{2}$, and $\epsilon_{3}$. Note that each porosity can take a value between zero and unity, because it is defined for the cascading representative solid volumes.

The predicted saturation currents, based on Eqs. 15 and 17, are also plotted for different values of $\epsilon_{1}$ in Figure 14. The results are also given in Table 1 . The measurements for $\epsilon_{1}=1$ (no diffusion barrier) show a good agreement with the predicted molecular diffusivity of oxygen. The results for the washcoat barrier are also in good agreement with the predictions of Eq. 17. In order to show the sensitivity of the predicted value of $\left\langle D_{m}\right\rangle$ to $\epsilon_{1}$ in Eq. 17, the saturation currents for $\epsilon_{1}=0.21$ (for the washcoat used in the experiment), and for $\epsilon_{1}=0.1$ and 0.3 , are shown in Figure 14. Large variations do occur among washcoat batches, so care must be taken to use the properties of the washcoats used in the experiments. The thickness of the washcoat plug in the experiment was not directly measured, and it is assumed to be the same as the stainless steel housing thickness.

\section{Conclusion}

The effects of arm randomness, arm thickness, and armthickness distribution on the effective diffusivity of porous media are predicted using 2-D network models. Predictions show that a random, tetragonal network model with a uniform arm-thickness distribution results in a lower limit for the effective diffusivity. However, the effects of the coordination number, lattice, and arm-thickness randomness on the effective diffusivity were found not to be very significant. Therefore, a simplified network model based on the random tetragonal (coordination number 4) network model with the normal arm-thickness distribution is suggested as the representative model. However, the arm thickness has a significant 


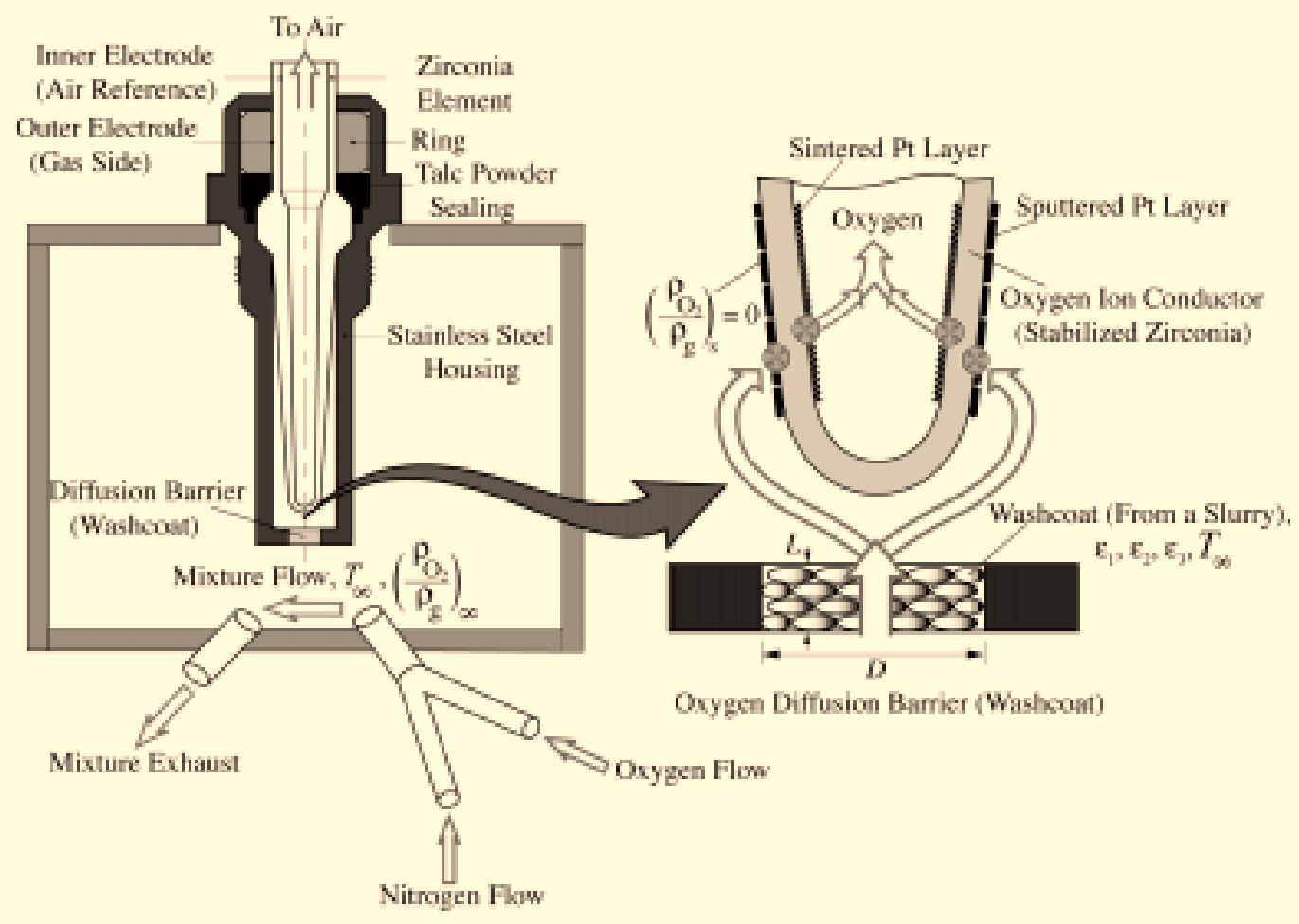

Figure 13. Effective diffusivity measurement device.

effect on the effective diffusivity and this effect is included in a modified molecular diffusivity relation, that is, Eq. 11.

The effect of the pore blockage (pore poisoning) is also correlated with the fraction of the volume occupied by the blocking contaminants, and along with the porosity dependence is given by Eq. 16. It should be pointed out that the generation of the poisonous contaminants assumed here is random, while in practice, there may be a preferential poisoning of the pores near the surface. Once these surface pores are blocked, there is no access to the inner pores. The effect of multiple pore length scales is given by the parallel model of diffusion by Eq. 17.

The 2-D network model used here creates pores that are not straight, but rather tortuous. It is customary to use this tortuosity as a geometric property of porous media. The effective diffusivity of porous media is then defined in terms of tortuosity $\tau$ and the constriction factor $\sigma$ as (Fogler, 1999)

$$
\left\langle D_{m}\right\rangle=D_{m}^{\prime} \frac{\epsilon \sigma}{\tau}
$$

The constriction factor represents the cross-sectional area change normal to the diffusion path, and is assumed to be unity. The tortuosity is defined as the ratio of the actual distance traveled by the gas molecules between two points to

Table 1. Parameters, Measured Quantities, and Deduced Variables in the $\mathrm{O}_{2}-\mathrm{N}_{2}$ Experiment

\begin{tabular}{|c|c|c|c|c|}
\hline $\begin{array}{l}D, \mathrm{~m} \\
L, \mathrm{~m} \\
T, \mathrm{~K} \\
a, \mathrm{~N}^{1 / 3} \cdot \mathrm{m}^{4 / 3} \cdot \mathrm{kg}^{1 / 2} / \mathrm{s} \cdot \mathrm{K}^{5 / 6} \cdot \mathrm{kmol}^{1 / 2} \\
b \\
\epsilon_{1} \\
\text { Predicted }\left\langle D_{m}\right\rangle, \mathrm{m}^{2} / \mathrm{s}\end{array}$ & & 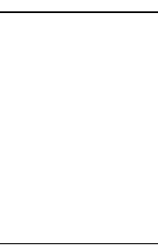 & & \\
\hline$\left(\rho_{\mathrm{O}_{2}} / \rho_{g}\right)_{\infty}$ & 0.05 & 0.10 & 0.05 & 0.10 \\
\hline$\rho_{\mathrm{O}_{2 \infty}}, \mathrm{kg} / \mathrm{m}^{3}$ & 0.0281 & 0.0562 & 0.0281 & 0.0562 \\
\hline$J_{e}, \mathrm{~mA}$ & $\begin{array}{l}77.0 \\
83.0 \\
84.1\end{array}$ & $\begin{array}{l}169.0 \\
165.0 \\
162.7\end{array}$ & $\begin{array}{l}25.4 \\
23.8 \\
24.6\end{array}$ & $\begin{array}{l}57.1 \\
55.5 \\
52.4\end{array}$ \\
\hline measured $\left\langle D_{m}\right\rangle, \mathrm{m}^{2} / \mathrm{s}$ & $\begin{array}{l}6.93 \times 10^{-5} \\
7.46 \times 10^{-5} \\
7.57 \times 10^{-5}\end{array}$ & $\begin{array}{l}7.60 \times 10^{-5} \\
7.42 \times 10^{-5} \\
7.32 \times 10^{-5}\end{array}$ & $\begin{array}{c}1.59 \times 10^{-5} \\
1.49 \times 10^{-5} \\
1.53 \times 10^{-5}\end{array}$ & $\begin{array}{l}1.78 \times 10^{-5} \\
1.73 \times 10^{-5} \\
1.64 \times 10^{-5}\end{array}$ \\
\hline
\end{tabular}




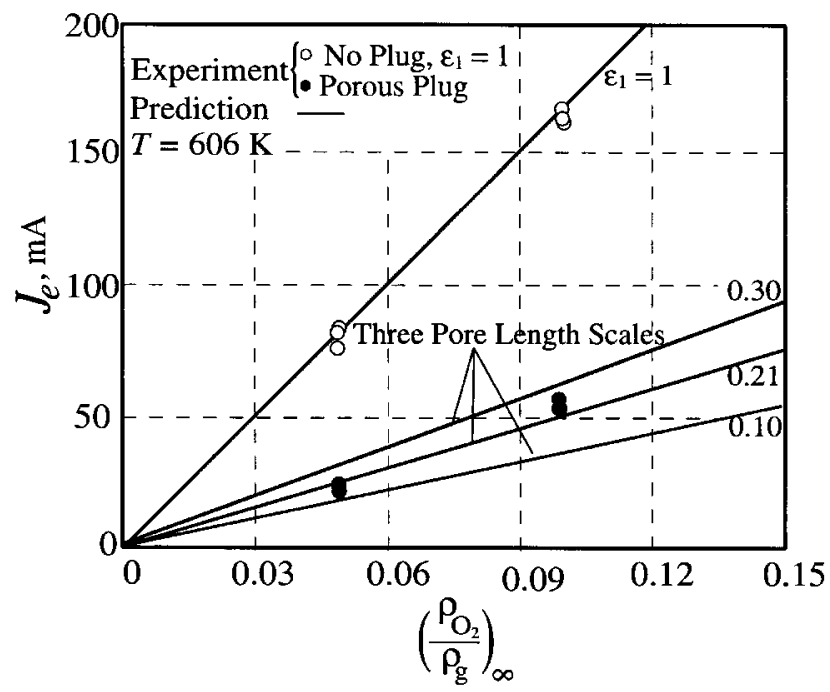

Figure 14. Measured saturation current for the washcoat plug (placed in an oxygen sensor) as a function of the far-field oxygen mass fraction.

The predicted saturation currents for several large length scale porosities are also shown.

the shortest distance between those two points. By combining Eq. 18 with Eq. 16, the effective tortuosity can be obtained.

The experiment performed on a multiple length scale washcoat layer showed a good agreement with the predictions.

The predicted effective diffusivity can be used in the analysis of simultaneous diffusion-reaction in porous media under various chemical and thermal nonequilibria (Oliveira and Kaviany, 2001). However, such network analysis should allow for simultaneous reaction along each of the length scale paths, each using the individual effective diffusivity $\left\langle D_{m}\right\rangle_{i}$.

\section{Acknowledgment}

The continued technical support of Dr. Amir A. M. Oliveira (currently at the Federal University of Santa Catarina, Brazil) is greatly appreciated. Mr. David Jordan assisted with the microscopy and characterization of the washcoat, as well as the network analysis. His contributions are also greatly appreciated.

\section{Notation}

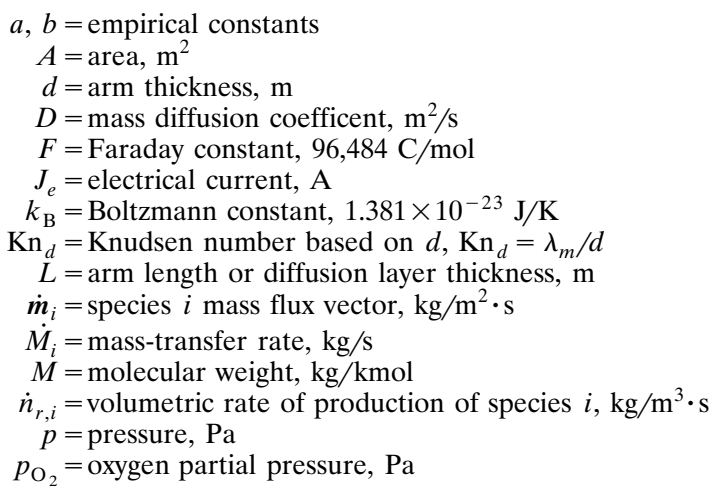

$R_{\mathrm{g}}=$ universal gas constant, $8.314 \times 10^{3} \mathrm{~J} / \mathrm{kmol} \cdot \mathrm{K}$

$T_{c}=$ critical temperature, $\mathrm{K}$

$T=$ temperature, $\mathrm{K}$

$\left\langle u_{m}^{2}\right\rangle^{1 / 2}=$ root-mean-square of thermal velocity, $\mathrm{m} / \mathrm{s}$

$V=$ volume $\mathrm{m}^{3}$

$\epsilon=$ porosity

$\tau=$ tortuosity

$\sigma=$ constriction factor

$\lambda_{m}=$ molecular mean-free path, $\mathrm{m}$

$\rho_{i}=$ density of species $i, \mathrm{~kg} / \mathrm{m}^{3}$

$\rho_{\mathrm{O}_{2}}=$ density, $\mathrm{kg} / \mathrm{m}^{3}$

$\Delta \varphi=$ electric potential difference, $\mathrm{V}$

$\Delta E_{a}=$ molar activation energy, $\mathrm{J} / \mathrm{kmol}$

\section{Subscripts}

$$
\begin{aligned}
b & =\text { blockage } \\
c & =\text { catalyst } \\
g & =\text { gas } \\
i, j & =\text { species } i \text { and } j \\
k, n & =\text { nodes } k \text { and } n \\
m & =\text { molecular } \\
\mathrm{K} & =\text { Knudsen } \\
p & =\text { pore } \\
s & =\text { surface } \\
s g & =\text { solid-gas interface } \\
\infty & =\text { far-field value }
\end{aligned}
$$

\section{Symbols}

$$
\begin{aligned}
\langle\rangle & =\text { volume or area averaged } \\
\prime & =\text { combined effect }
\end{aligned}
$$

\section{Literature Cited}

Bird, R., E. Stewart, and N. Lightfoot, Transport Phenomena, Wiley, New York (1960).

Cunningham, R. E., and R. J. J. Williams, Diffusion in Gases and Porous Media, Plenum Press, New York (1980).

Fogler, H. S., Elements of Chemical Reaction Engineering, 3rd ed., Prentice-Hall, Upper Saddle River, NJ (1999).

Hollewand, M. P., and L. F. Gladden, "Modeling of Diffusion and Reaction in Porous Catalysts Using a Random Three-Dimensional Network Model," Chem. Eng. Sci., 47, 1761 (1992).

Kast, W., and C.-R. Hohenthanner, "Mass Transfer within the Gas Phase of Porous Media," Int. J. of Heat and Mass Transf., 43, 807 (2000).

Kaviany, M., Principles of Heat Transfer, Wiley, New York (2001).

Kaviany, M., Principles of Heat Transfer in Porous Media, 2nd ed., Springer-Verlag, New York (1995).

Kim, B. K., J. H. Lee, and H. Kim, "A Wide Range Air-Fuel Ratio Sensor With a Plate Type Structure," Solid State Ionics, 86-88, 1079 (1996).

Lee, J. H., C. S. Kwon, H. Kim, and B. K. Kim, "Limiting Current and Wide Range Air/Fuel Ratio Sensor Using the Same YSZ$\mathrm{Al}_{2} \mathrm{O}_{3}$ Layers as Electrolyte and Diffusion Barrier," Sensors and Actuators B, 35-36, 278 (1996).

Mann, R., J. J. Almeida, and M. N. Mugerwa, "A Random Pattern Extension to the Stochastic Network Pore Model," Chem. Eng. Sci., 41, 2663 (1986).

Neale, G. H., and W. K. Nader, "Prediction of Transport Processes within Porous Media: Diffusive Flow Processes within Homogeneous Swarms of Spherical Particles," AIChE J., 19, 112 (1973).

Ohya, T., M. Iri, and K. Murota, "A Fast Voronoi-Diagram Algorithm with Quaternary Tree Bucketing," Inf. Process. Lett., 18, 227 (1984).

Oliveira, A. A. M., and M. Kaviany, "Nonequilibrium in the Transport of Heat and Reactants in Combustion in Porous Media," Prog. Energy Comb. Sci., 27, 523 (2001).

Weisz, P. B., "Zeolites-New Horizons in Catalysis," Chemtec, 8, 498 (1973).

Manuscript received Nov. 30, 2000, and revision received July 5, 2001. 